\title{
Influence of tourniquet application on venous blood sampling for serum chemistry, hematological parameters, leukocyte activation and erythrocyte mechanical properties
}

\author{
Melike Cengiz ${ }^{1,2}$ *, Pinar Ulker ${ }^{2}$, Herbert \\ J. Meiselman ${ }^{3}$ and Oguz K. Baskurt ${ }^{2}$ \\ ${ }^{1}$ Department of Anesthesiology and ICU, Akdeniz \\ University Faculty of Medicine, Antalya, Turkey \\ ${ }^{2}$ Department of Physiology, Akdeniz University \\ Faculty of Medicine, Antalya, Turkey \\ ${ }^{3}$ Department of Physiology and Biophysics, Keck \\ School of Medicine, University of Southern \\ California, Los Angeles, CA, USA
}

\begin{abstract}
Background: Venous blood sampling is usually performed using a tourniquet to help locate and define peripheral veins to achieve successful and safe venipuncture. Despite widespread usage of tourniquets for venipuncture by medical and laboratory staff, very few are aware of the effects of tourniquet application on laboratory parameters. In addition, definitive guidelines regarding when and how to use a tourniquet for blood sampling are lacking. The aim of the present study was to define the optimal sampling time after tourniquet removal to avoid adverse impact on laboratory analytes.
\end{abstract}

Methods: Blood oxygen and carbon dioxide partial pressure, $\mathrm{pH}$, oxyhemoglobin saturation $\left(\mathrm{satO}_{2}\right)$, hematological parameters, serum electrolyte concentrations, erythrocyte, deformability and aggregation, leukocyte activation and nitrite/nitrate concentrations obtained $180 \mathrm{~s}$ after tourniquet release were compared with baseline values for 10 healthy subjects.

Results: Blood gases, hematological parameters and serum electrolyte levels were not affected by the application and removal of a tourniquet. However, there were significant decreases in erythrocyte deformability at $90,120,180 \mathrm{~s}$, and increases in erythrocyte aggregation at 5 and $30 \mathrm{~s}$ following removal of the tourniquet. A significant increase in granulocyte respiratory burst at $60 \mathrm{~s}$ was observed, confirming leukocyte activation due to application of the tourniquet. There were no significant alterations of blood nitrite/nitrate levels.

Conclusions: Our blood sampling technique which mimicked the application and release of a tourniquet indicated unaltered values for routine blood gases, hematological testing and serum electrolyte levels. Conversely, hemorheological measurements can be

*Corresponding author: Melike Cengiz, MD, Department of Anesthesiology and ICU, Hospital of Akdeniz University, 07070 Antalya, Turkey

Phone: +90 2422496246, Fax: +90 2422278836,

E-mail: melikecengiz@yahoo.com

Received December 29, 2008; accepted March 24, 2009; previously published online May 11, 2009 affected. Therefore, it is strongly recommended that tourniquet application should be avoided during blood sampling or, if this is not possible, the procedure should be well standardized and details of the sampling method should be reported.

Clin Chem Lab Med 2009;47:769-76.

Keywords: blood sampling; hemorheology; leukocyte activation; tourniquet application.

\section{Introduction}

A tourniquet is widely used during blood sampling for facilitating visualization of the vein and to perform successful venipuncture. Most of the studies in this area have investigated changes in hematologic, biochemical, coagulation and rheologic properties of venous blood at different time points following tourniquet application, but these results cannot be easily adapted to a single standard procedure (1-4). Evidence from previous studies is that tourniquet application before blood sampling may affect some laboratory parameters $(1,4,5)$. However, although the use of a tourniquet for blood sampling has been discussed in the literature (6-8), there still is lack of definitive information indicating the most appropriate timing for blood sampling when a tourniquet is used.

In routine practice, blood samples are usually obtained without loosening the tourniquet. Thus, blood withdrawal is very rapid and maintaining the needle inside the vessel lumen is made easier due to venous stasis. Alternatively, it has been recommended that for hemorheological studies, the tourniquet should be removed at least $5 \mathrm{~s}$ prior to the sampling of blood (9). However, a recent study indicated that significant alterations may be detected in red blood cell (RBC) deformability and aggregation 5-90 s after the removal of tourniquet (10). Surprisingly, RBC deformability was found to be increased (10), thus suggesting a role for nitric oxide (NO) in the alteration of RBC deformability (11).

The aim of the present study was to investigate the magnitude and duration of changes in blood gases, hematological parameters, serum electrolyte concentrations, hemorheological parameters, nitrite/nitrate content and granulocyte burst activity in blood samples obtained shortly after the restoration of venous flow following a brief occlusion model of tourniquet application. Blood sampling, including simulated tourniquet application, was performed with blood samples obtained consecutively for $3 \mathrm{~min}$ after restoration of venous blood flow. The primary goal was 
to define the optimal sampling time following tourniquet removal that did not affect some of the widely used clinical laboratory and hematological/hemorheological parameters.

\section{Materials and methods}

\section{Study design}

Healthy male subjects $(n=10)$, aged between $25-30$ years, were included in this study. Written informed consent was obtained and the study was approved by the local Ethics Committee. The venipuncture procedure for all subjects was performed by an anesthetist with the subject in a sitting position. A $20 \mathrm{G}$ catheter (BD Venflon ${ }^{\mathrm{T}}{ }^{\text {, }}$, Pro Safety, Becton Dickinson, Helsingborg, Sweden) was inserted into the right antecubital vein using a tourniquet. The tourniquet was removed following insertion of the catheter. After insertion of the catheter and removal of the tourniquet, a 30-min waiting period was observed to allow for stabilization. Following stabilization, blood pressure in the left arm was measured and diastolic pressure determined (Figure 1). Control blood samples were obtained from the catheter as described below (see Collection of blood samples). A pneumatic cuff was then applied to the right arm above the previously inserted catheter and venous stasis was imposed for $30 \mathrm{~s}$ by inflating the cuff at a pressure $20 \mathrm{~mm} \mathrm{Hg}$ higher than diastolic blood pressure (DBP, see next paragraph). The cuff was then deflated to mimic removal of a tourniquet, and blood samples were obtained from the catheter at $5,30,60,90,120,150,180 \mathrm{~s}$ following deflation of the cuff. Blood oxygen and carbon dioxide partial pressures $\left(\mathrm{pO}_{2}\right.$ and $\left.\mathrm{pCO}_{2}\right), \mathrm{pH}$, oxyhemoglobin saturation $\left(\mathrm{satO}_{2}\right.$ ) hematological parameters, serum electrolyte concentrations, RBC deformability and aggregation, and nitrite/nitrate concentrations were measured in all blood samples. However, granulocyte respiratory burst was determined only in the blood samples obtained at baseline (control) and at 60 and $180 \mathrm{~s}$ following cuff deflation.

The use of a cuff pressure $20 \mathrm{~mm} \mathrm{Hg}$ above DBP was determined based upon a pilot study performed at the Akdeniz University Intensive Care Unit using a patient having a radial artery catheter inserted for blood pressure monitoring. Eight physicians or nurses, who were unaware of the study protocol, were asked to apply an elastic tourniquet on the patients arm with the arterial catheter. The arterial pressure wave shape observed on a monitor was deformed, but did not disappear after the tourniquet application. The same type of deformation was observed when a pneumatic cuff was applied and gradually inflated to the range of mean arte-

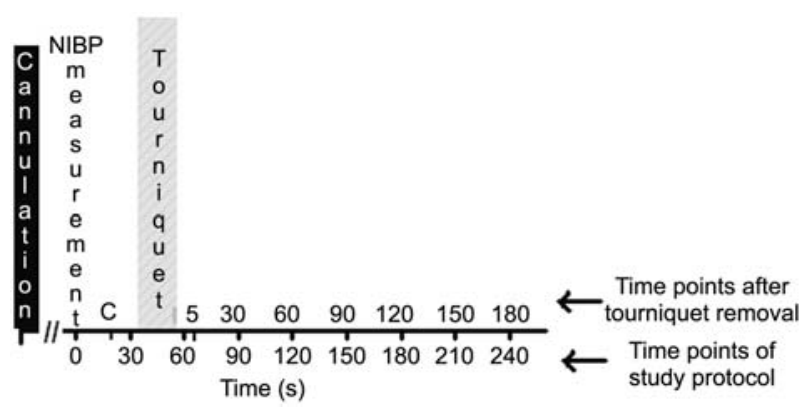

Figure 1 Experimental protocol and blood sampling times. The study was initiated with blood pressure measurement followed by blood sampling at defined times after tourniquet (i.e., pneumatic cuff) removal. NIBP, non-invasive blood pressure; C, control sample. rial pressure for this patient (i.e., systolic blood pressure of 110-120 mm Hg, DBP of 55-60 mm Hg). Therefore, a cuff pressure ranging between systolic pressure and $20 \mathrm{~mm} \mathrm{Hg}$ above DBP was selected for modelling application of the tourniquet. It should be mentioned that this standardized procedure resulted in a total occlusion of venous flow during the inflation of the cuff, but arterial flow was not abolished.

\section{Collection of blood samples}

Two separate blood samples were obtained at each time point:

1. Eight milliliter was collected at control sampling, and at 60 and $120 \mathrm{~s}$ following the deflation of the cuff. Four milliliter of blood was obtained at all other time points. Blood was collected into a syringe containing sodium heparin $(15 \mathrm{IU} / \mathrm{mL})$. These anticoagulated blood samples were used for analysis of blood $\mathrm{pO}_{2}, \mathrm{pCO}_{2}, \mathrm{pH}$, satO ${ }_{2}$, measurement of RBC deformability and aggregation, quantification of granulacyte respiratory burst and nitrite/ nitrate concentration. Processing of the specimens was performed immediately after blood collection except for quantitation of nitrite/nitrate concentrations. Plasma was refrigerated $\left(-20^{\circ} \mathrm{C}\right)$ and used for quantitation of nitrite/ nitrate following collection of specimens from all study subjects.

2. Four milliliter of blood was obtained using a syringe not containing anticoagulant, and immediately divided into two tubes: a) a $K_{2}$ EDTA coated tube (BD Vacutainer Systems, Preanalytical Solutions, Belliver Industrial Estate, Plymouth, UK) for analysis of hematological parameters. b) a serum separator tube containing gel to separate serum from RBC following centrifugation (Greiner BioOne/VACUETTE ${ }^{\circledR}$, Longwood, FL, USA). This was used for analysis of serum electrolytes. Samples were analysed within $1 \mathrm{~h}$ after collection.

\section{Analysis of blood $\mathrm{pO}_{2}, \mathrm{pCO}_{2}, \mathrm{pH}$, satO ${ }_{2}$, hematological parameters and serum electrolyte concentrations}

Analysis of blood $\mathrm{pH}$, partial carbon-dioxide pressure $\left(\mathrm{pCO}_{2}\right)$, partial oxygen pressure $\left(\mathrm{pO}_{2}\right)$, oxygen saturation $\left(\mathrm{satO}_{2}\right)$, bicarbonate $\left(\mathrm{HCO}_{3}{ }^{-}\right)$content and base excess (BE) was performed by using a Stat Profile CCX blood gas analyzer (Nova Biomedical, Waltham, MA, USA).

RBC count, white blood cell (WBC) count, hemoglobin, hematocrit, mean corpuscular volume (MCV), mean corpuscular hemoglobin concentration (MCHC), platelets, lymphocytes and neutrophils were assayed using a Beckman Coulter LH 780 (Beckman Coulter Inc, Fullerton, CA, USA). Serum sodium, potassium, chloride, calcium and magnesium were measured on a Roche/Hitachi Modular System P (Roche Diagnostics $\mathrm{GmbH}$, Mannheim, Germany).

\section{RBC deformability measurements}

RBC deformability was measured as an elongation index (EI) at $37^{\circ} \mathrm{C}$ for various fluid shear stresses by laser diffraction analysis using an ektacytometer (LORRCA, RR Mechatronics, Hoorn, The Netherlands). The principle of the system has been described elsewhere in detail (12). Briefly, a low hematocrit suspension of RBC in an isotonic viscous medium (4\% polyvinylpyrrolidone 360 solution; MW $360 \mathrm{kDa}$ ) is sheared in a Couette system composed of a glass cup and a precisely fitting bob, with a gap of $0.3 \mathrm{~mm}$ between the cylinders. A laser beam is directed through the sample and the diffraction 
pattern produced by the deformed cells is analyzed with a microcomputer. Based upon the geometry of the elliptical diffraction pattern, an $E I$ is calculated as: $E I=(L-W) / L+W)$, where $L$ and $W$ are the length and width of the diffraction pattern. At a constant shear stress, El increases with RBC deformability.

El values determined at the nine shear stress between 0.3-30 pascal $(\mathrm{Pa})$ were used to calculate the shear stress required for half-maximal $\mathrm{RBC}$ deformation $\left(\mathrm{SS}_{1 / 2}\right)$ using Lineweaver-Burk analysis as described elsewhere (13). Briefly, shear stress - El curves were linearized by plotting the reciprocal of $\mathrm{EI}$ as the function of the reciprocal of shear stress. The $x$-intercept of the line obtained by simple linear regression corresponds to the negative reciprocal value of shear stress causing $\mathrm{SS}_{1 / 2}$. Thus, increased $\mathrm{SS}_{1 / 2}$ values indicate impairment of RBC deformability.

\section{RBC aggregation measurements}

RBC aggregation was assessed using a custom-built photometric aggregometer, interfaced to a digital computer, that monitored light transmittance through the blood sample during the aggregation process (14). This device was used instead of the aggregation mode of the LORCA due to the smaller sample size $(0.1 \mathrm{~mL}$ for aggregometer vs. $1 \mathrm{~mL}$ blood required for the LORCA). The shearing portion of the system consists of two parallel glass plates with a gap of $0.3 \mathrm{~mm}$ between them. A stepper motor, controlled by the computer, rotates one of these plates. The blood sample under investigation is placed between the glass plates, and is first sheared at $500 \mathrm{~s}^{-1}$ for $10 \mathrm{~s}$ to disperse pre-existing $\mathrm{RBC}$ aggregates. Following a sudden stop of the motor, the infrared light transmission through the blood sample is monitored for $10 \mathrm{~s}$, integrated by the computer, and a dimensionless index calculated that increases with the extent of RBC aggregation. Measurements were performed in triplicate for each sample, and the mean of the three measurements used as the result. RBC aggregation was measured for cells in autologous plasma and for cells resuspended in isotonic phosphate buffer saline (PBS) (290 $\mathrm{mOsm} / \mathrm{kg}, \mathrm{pH}=7.4$ ) containing $0.5 \%$ dextran 500 (500 kDa, Sigma Chemical Co, St Louis, CA, USA) to determine the intrinsic tendency of RBCs to aggregate (15). Measurements were carried out at $37^{\circ} \mathrm{C}$ with the hematocrit of all samples adjusted to $0.4 \mathrm{~L} / \mathrm{L}$.

\section{Quantification of granulocyte respiratory burst}

The principle of the method has been described elsewhere (16). Briefly, granulocytes were isolated from whole blood using polysucrose density gradients (Histopaque 1119; Sigma Chemical Co.) as described previously (17). Isolated granulocytes were washed with and suspended in PBS at a cell count of $2.5 \times 10^{6} / \mathrm{mL}$. The granulocyte suspension was then incubated in the dark at $37^{\circ} \mathrm{C}$ for $25 \mathrm{~min}$ in the presence of $10^{-6} \mathrm{~mol} / \mathrm{L} \mathrm{2}{ }^{\prime}, 7^{\prime}$-dichlorofluorescrein diacetate (Catalogue No: D-6883; Sigma Chemical Co.). The fluorescence of dichlorofluorescrein diacetate was determined in granulocyte suspensions $\left(10^{6} / \mathrm{mL}\right.$ PBS) using a Perkin Elmer LS-50 spectrofluorometer (Spectral Genomics, Boston, MA, USA) with $335 \mathrm{~nm}$ excitation and $655 \mathrm{~nm}$ emission wavelengths, the intensity of the fluorescence being directly related to the degree of activation of granulocytes, and therefore, granulocyte respiratory burst. Preparation of each specimen for quantitation of granulocyte respiratory burst took $1 \mathrm{~h}$. Since every specimen was prepared in the same manner, the results were assumed to reflect the activation status of granulocytes at the time of specimen collection.

\section{Quantification of nitrite/nitrate concentration}

Nitrite/nitrate concentration in RBC suspensions was quantified using a colorimetric kit (No: 78001, nitrite/nitrate calorimetric assay kit; Cayman Chemical, Ann Arbor, MI, USA); the principle of the method has been previously described (18). Blood samples were centrifuged at $800 \mathrm{~g}$ for $6 \mathrm{~min}$ to isolate plasma. Proteins were removed from plasma samples by centrifugation at $100,000 \mathrm{~g}$ for $30 \mathrm{~min}$ at $4^{\circ} \mathrm{C}$. Following centrifugation, the plasma was transferred to a $10-\mathrm{kDa}$ cut-off filter (Millipore Corporation, Billerica, MA, USA) and re-centrifuged at $18,000 \mathrm{~g}$ for $120 \mathrm{~min}$ at $4^{\circ} \mathrm{C}$. The resultant fluid was incubated with nitrate reductase and its cofactor at room temperature $\left(20-22^{\circ} \mathrm{C}\right)$ for $3 \mathrm{~h}$. After a second incubation with Griess reactants $\left(R_{1}, R_{2}\right)$ for 10 min, absorbance was measured with a Thermo Labsystems multiscan, spectrum-1500 spectrophotometer (Thermo Fisher Scientific, Waltham, MA, USA) at $540 \mathrm{~nm}$. Nitrite/nitrate concentrations were calculated using a standard curve.

\section{Statistical analysis}

Values are presented as mean $\pm \mathrm{SE}$ of the mean. One-way analysis of variance (ANOVA) was used for repeated measures followed by Dunnetts Multiple Comparison post-hoc test. The level of statistical significance was set at $p<0.05$. Analysis was performed using Graphpad Prism IV software (GraphPad Software Inc, La Jolla, CA, USA).

\section{Results}

\section{Blood $\mathrm{pO}_{2}, \mathrm{pCO}_{2}, \mathrm{pH}$, satO $\mathrm{S}_{2}$, hematological parameters and serum electrolyte concentrations}

Blood $\mathrm{pO}_{2}, \mathrm{pCO}_{2}$, satO ${ }_{2}$ and $\mathrm{HCO}_{3}{ }^{-}$values were not different in the samples obtained at $30 \mathrm{~s}$ intervals during the $3 \mathrm{~min}$ period following the restoration of venous flow. However, blood $\mathrm{pH}$ values were found to be decreased at 5 and $30 \mathrm{~s}$ following deflation of the pneumatic cuff. Values returned to baseline afterwards (Table 1).

Hematological parameters including RBC, WBC, platelet, lymphocyte and neutrophil counts, hemoglobin, hematocrit, MCV and MCHC were not significantly altered in any of the samples following cuff deflation (Table 2). Serum sodium, potassium, calcium and magnesium levels were also unaffected (Table 3).

\section{RBC deformability}

$\mathrm{SS}_{1 / 2}$ values were found to be significantly increased at 90,120 and $180 \mathrm{~s}$ after the pneumatic cuff was deflated but were not affected at earlier time points (Table 4). This indicated impaired RBC deformability.

\section{RBC aggregation}

RBC aggregation measured in autologous plasma was found to be increased significantly at $5(p<0.05)$ and $30 \mathrm{~s}(\mathrm{p}<0.01)$ after release of the pneumatic cuff. Values returned to baseline after $120 \mathrm{~s}$ (Table 4).

RBC aggregation measured in isotonic PBS containing $0.5 \%$ dextran 500 was not altered in any of the blood samples obtained after removal of the pneumatic cuff ( $p>0.05)$ (Table 4$)$. 

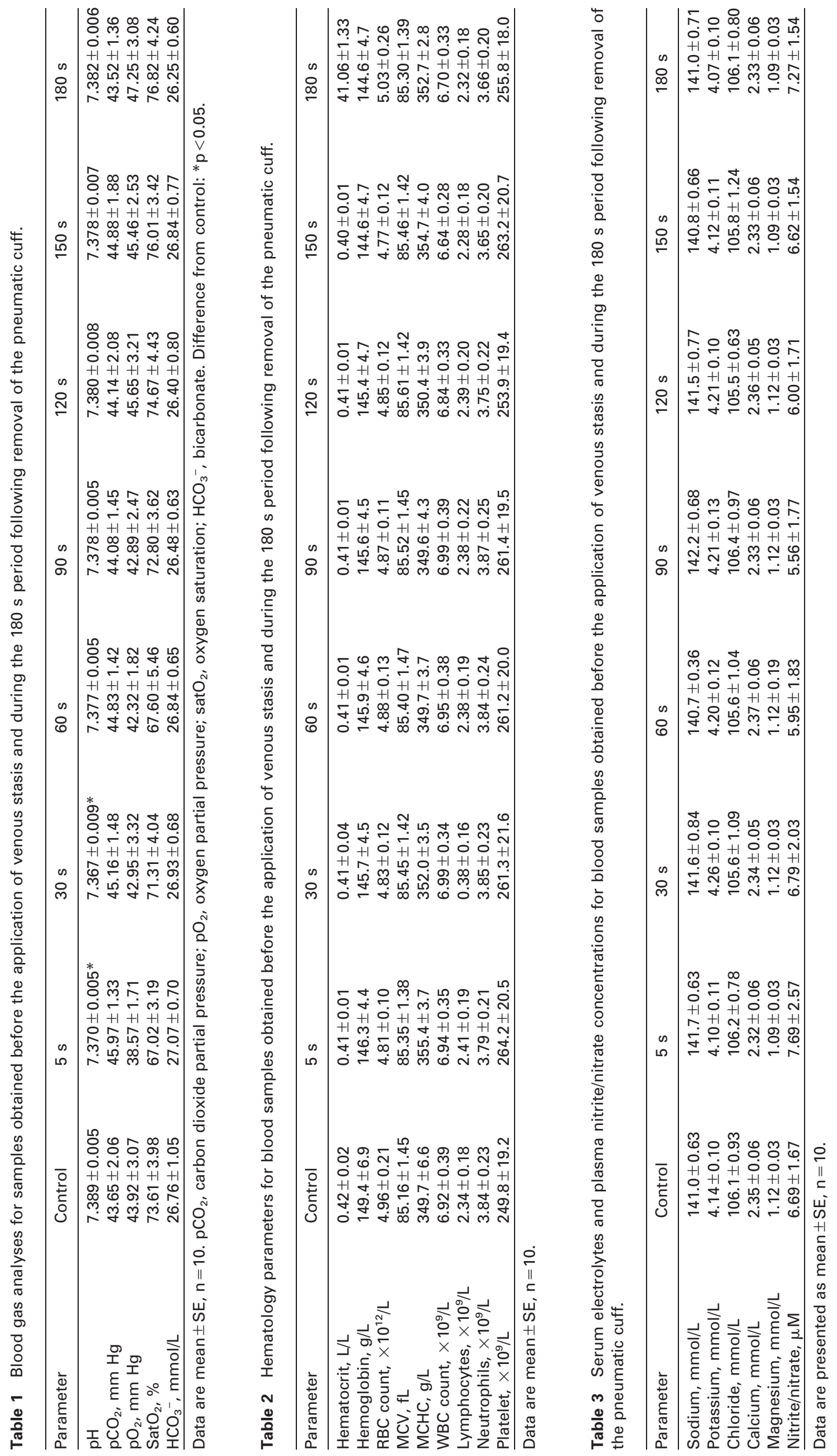


\section{Granulocyte respiratory burst}

Granulocyte respiratory burst increased at $60 \mathrm{~s}$ $(p<0.01)$ and returned to baseline values at $180 \mathrm{~s}$ $(p>0.05)$ following deflation of the cuff (Figure 2).

\section{Nitrite/nitrate concentration}

Plasma nitrite/nitrate concentrations did not change at any sampling time after deflation of the pneumatic cuff $(p>0.05)$ (Table 3 ).

\section{Discussion}

The results of the present study indicate that blood gases, hematological parameters and serum electrolyte concentrations were not significantly affected by temporary venous stasis when using a pneumatic cuff as a model for tourniquet use during blood sampling. These findings are in contrast with previous reports $(1,2)$. However, there were significant decreases in $\mathrm{RBC}$ deformability and increases in RBC aggregation following release of the pneumatic cuff. Granulocyte respiratory burst was significantly increased at $60 \mathrm{~s}$ after the restoration of venous flow, indicating activation following a short $30 \mathrm{~s}$ period of venous stasis. Finally, restoration of venous flow following $30 \mathrm{~s}$ of stasis did not cause significant alterations in blood nitrite/nitrate concentrations.

A decrease in $\mathrm{pO}_{2}$ during tourniquet application that occludes venous return for $10 \mathrm{~min}$ has been previously reported (4). However, this $10 \mathrm{~min}$ duration is 20 -fold longer than the $30 \mathrm{~s}$ used in this study. It should be noted that blood flow was restored before the start of sampling for both the current and previous study (4). The previous study reported a return of blood gas levels to baseline concentrations 60 s postocclusion. This finding is consistent with our results at $60 \mathrm{~s}$ (Table 1). Blood pH was found to be slightly decreased at 5 and $30 \mathrm{~s}$ after cuff deflation (Table 1).

No significant changes in serum concentrations of sodium, potassium, chloride, calcium and magnesium were observed after release of the cuff. Renoe et

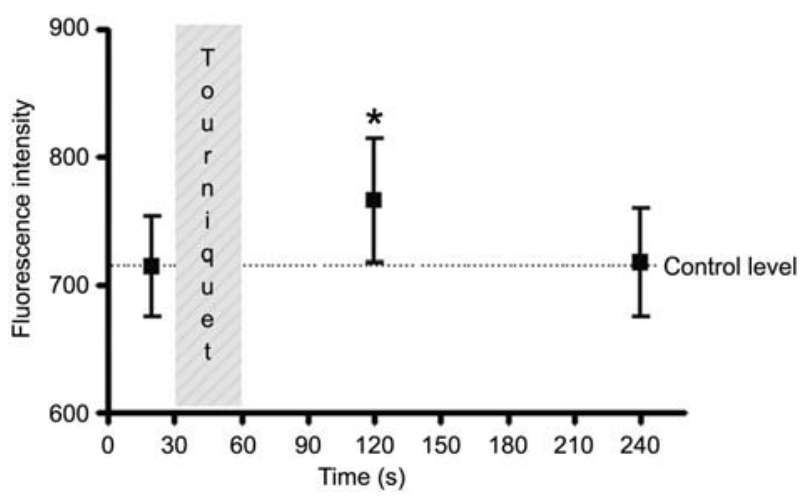

Figure 2 Granulocyte activation (i.e., respiratory burst) prior to and after release of pressure cuff.

Fluorescence intensity was increased at $120 \mathrm{~s}$ and returned to control at $240 \mathrm{~s}$. Data are mean $\pm \mathrm{SE}, \mathrm{n}=10$. Difference from control; ${ }^{*} \mathrm{p}<0.05$. 
al. observed increased serum calcium concentrations at 1 min following the application of a pneumatic cuff (19). However, the duration of stasis was 3 min compared to $30 \mathrm{~s}$ used in this study. Furthermore, the effect of stasis was enhanced if the arm used for sampling was exercised. Continuous occlusion of venous flow following tourniquet use can alter electrolyte concentrations. Lippi et al. (2) report a significant decrease in potassium and a significant increase in calcium after $1 \mathrm{~min}$ of occlusion, a decrease of chloride at $3 \mathrm{~min}$ and unchanged sodium at both 1 and 3 min. However, Lippi et al. (2) obtained samples during venous stasis and not following restoration of blood flow.

Hematological parameters were not influenced by the $30 \mathrm{~s}$ period of venous stasis, regardless of the sampling following restoration of blood flow. The discrepancy between the results of the current study and the data from the previous studies exploring the effects of tourniquet application on clinical chemistry and hematological parameters may result from differences in the duration of venous stasis (tourniquet application) between studies (1,2). Previous studies were performed with venous stasis following the application of a standardized external pressure of $60 \mathrm{~mm} \mathrm{Hg}$ for 1 and $3 \mathrm{~min}$. The external pressure was maintained until venipuncture and collection of blood were completed. However, in the current study, venous stasis was imposed for $30 \mathrm{~s}$ by inflating the cuff at a pressure $20 \mathrm{~mm} \mathrm{Hg}$ higher than DBP, and blood samples were collected following deflation of the cuff.

Hemorheological changes after venous stasis, with or without arterial occlusion, have been reported previously $(4,5)$, with impairment of RBC deformability generally consistent with our findings. In the study by Forconi (4), whole blood filtration returned to baseline values in samples that were withdrawn 1 min after the release of cuff pressure. Subsequent measurements were not performed. However, in the current study, changes in RBC deformability started $90 \mathrm{~s}$ after restoration of venous flow, and did not return to baseline values at $3 \mathrm{~min}$. In contrast, a recent study showed that RBC deformability was improved, rather than impaired, following removal of the tourniquet, with the alterations that were observed at $60 \mathrm{~s}$ no longer evident at $90 \mathrm{~s}(10)$. The discrepancy between the present study and the recent report (10) is most likely due to different approaches to stop blood flow. The pressure applied in the current study was welldefined (i.e., $20 \mathrm{~mm} \mathrm{Hg}$ above diastolic), and thus was sufficient to occlude venous blood flow but not stop arterial inflow. Based upon the results of pilot studies to determine the appropriate pressure (see Study design above), we believe that our approach represents a more realistic model of tourniquet use during blood collection.

RBC aggregation in blood samples obtained at 5 and $30 \mathrm{~s}$ after restoration of venous flow was found to be increased in autologous plasma, but not in the standard $0.5 \%$ dextran 500 aggregating medium (Table 4). These findings indicate that cellular factors contributing to RBC aggregation were not altered due to venous stasis. Rather, alterations in plasma composition (e.g., increased concentration of fibrinogen resulting from local fluid shifts) may be responsible for enhanced aggregation in plasma. Our findings of no changes between 60 and $180 \mathrm{~s}$ are in contrast to those of a previous report (10) which showed a decrease in RBC aggregation $90 \mathrm{~s}$ after the restoration of blood flow. As in previous studies different approaches used to stop blood flow may be responsible for these differences.

The mechanisms causing alterations in the parameters we studied following tourniquet application are not well known. Thus, we considered two factors that may be responsible for these changes.

(1) Neutrophil activation. Venous stasis may affect the number of marginated leukocytes and the number of blood cells adhering to venous endothelium. Leukocyte activation may increase with decreased venous blood flow velocity and shear rate (20). Also blood stasis and venous hypertension may lead to release of inflammatory mediators and growth factors that trigger the process of leukocyte recruitment and activation, migration, rolling and infiltration of the vessel wall (21). The results of the current study revealed an increase in granulocyte respiratory burst at $60 \mathrm{~s}$ which returned to baseline values at $180 \mathrm{~s}$. It has been previously reported that activated leukocytes induce structural and functional alterations in neighboring $\mathrm{RBC}$, including increased membrane lipid peroxidation and cell lysis, changes in RBC membrane skeletal proteins (e.g., cross-linking between spectrin and hemoglobin), and decreased RBC deformability (22-24). RBC aggregation has also been found to be enhanced by activated neutrophils, with the increase mediated by both proteolytic enzymes and oxygen free radicals (24). Thus the alterations of RBC deformability and aggregation in the current study may have resulted from leukocyte activation induced by venous stasis.

(2) Nitric oxide generation. NO is a well-known vasoactive mediator which regulates vascular tone, platelet aggregation, coagulation, fibrinolysis and leukocyte activation (25). Release of NO from vascular endothelium may be influenced by wall shear stress, intravascular stasis and ischemia-reperfusion injury $(26,27)$. In a previous study, plasma nitrate concentration was found to be significantly higher 5 min after reperfusion, compared to concentrations prior to ischemia (28). NO has also been shown to play a significant role in the regulation of RBC mechanical properties $(11,29)$, with a biphasic effect dependent on NO concentration (11). In addition to NO from external sources, NO generated within RBC has been suggested to influence deformability, based on observations related to significant effects of nitric oxide synthase (NOS) inhibitors (11). Recent studies have documented the activity of NOS in RBC. This NOS has properties similar to endothelial NOS $(29,30)$.

It has been suggested that NO may play a protective role in various patho-physiological processes characterized by impaired microcirculatory perfusion 
(e.g., ischemia-reperfusion injury, sepsis, sickle-cell disease) (30). Therefore, it is tempting to suggest that alterations of NO generation may have played a role in hemorheological alterations following restoration of blood flow. However, in the current study, no significant change in the concentration of plasma nitrite/ nitrate was observed, thereby excluding this possibility. Nevertheless, this finding should be interpreted cautiously as methods more sensitive than the Griess assay, which measures nitrite/nitrate concentration in the $\mathrm{nM}$ range, could indicate changes in NO activity (31).

In this study, blood samples were obtained from a catheter to collect serial blood samples and avoid multiple attempts at venipuncture after the release of the tourniquet. However, use of catheters to collect routine samples is not the normal procedure and this may influence several laboratory parameters. Extrapolating our findings to the design of routine blood collection procedures using needles should be one with caution. Another limitation of the current study was the lack of a parameter to evaluate platelet function. Platelet function might be changed after tourniquet release since shear stress is an important activator of platelets.

In conclusion, the results of this study indicate that blood sampling for the measurement of blood gases, hematological parameters or serum electrolyte concentrations can be performed with the application of a tourniquet for $30 \mathrm{~s}$ without any adverse effects on these measured parameters. However, hemorheological changes do occur following application of a tourniquet. Therefore, it is strongly recommended that tourniquet application should be avoided during blood collection for the investigation of hemorheological parameters. If this is not possible, the procedure should be standardized and details of the sampling method should be reported.

\section{Acknowledgements}

This study was supported by NIH Research Grants HL15722, HL 70595 and FIRCA IR03 TW01295, and by the Akdeniz University Research Projects Unit.

\section{References}

1. Lippi G, Salvagno GL, Montagnana M, Franchini M, Guidi GC. Venous stasis and routine hematologic testing. Clin Lab Haem 2006;28:332-7.

2. Lippi G, Salvagno GL, Montagnana M, Brocco G, Guidi GC. Influence of short-term venous stasis on clinical chemistry testing. Clin Chem Lab Med 2005;43:869-5.

3. Lippi G, Salvagno GL, Montagnana M, Guidi GC. Shortterm venous stasis influences routine coagulation testing. Blood Coagul Fibrinolysis 2005;16:453-8.

4. Forconi S. Hemorheological changes during venous stasis as result of tourniquet application. Clin Hemorheol Microcirc 1999;20:197-9.

5. Rosenson RS, Tangney CC. Effects of tourniquet application on plasma viscosity measurements. Clin Hemorheol Microcirc 1998;18:191-4.
6. Junge B, Hoffmeister H, Feddersen HM, Rocker L. Standardisation of obtaining blood samples: influence of tourniquet application on 33 constituents of blood and serum. Dtsch Med Wochenschr 1978;103:260-5.

7. Thompson JM. Blood collection and preparation: preanalytical variation. In: Jesperson J, Bertina RM, Haverkate $F$, editors. ECAT assay procedure. A manual of laboratory techniques. Dordrecht: Kluwer Academic Publishers, 1992:13-20.

8. McNair P, Nielsen SL, Christiansen C, Axelsson C. Gross errors made by routine blood sampling from two sites using a tourniquet applied at different positions. Clin Chim Acta 1979;98:113-8.

9. ECSH Expert Panel on Blood Rheology, Guidelines for Measurement of Blood-Viscosity and Erythrocyte Deformability. Clin Hemorheol 1986;6:439-53.

10. Connes P, Uyuklu M, Tripette J, Boucher JH, Beltan E, Chalabi T, et al. Sampling time after tourniquet removal affects erythrocyte deformability and aggregation measurements. Clin Hemorheol Microcirc 2009;41:9-15.

11. Bor-Kucukatay M, Wenby RB, Meiselman HJ, Baskurt OK. Effects of nitric oxide on red blood cell deformability. Am J Physiol Heart Circ Physiol 2003;284:H1577-84.

12. Hardeman MR, Goedhard PT, Dobbe JGG, Lettinga KP. Laser-assisted optical rotational cell analyzer (LORCA). 1. A new instrument for measurement of various structural hemorheological parameters. Clin Hemorheol 1994;14: 605-18.

13. Baskurt OK, Meiselman HJ. Analyzing shear stress-elongation index curves: comparison of two approaches to simplify data presentation. Clin Hemorheol Microcirc 2004;31:23-30.

14. Baskurt OK, Meiselman HJ, Kayar E. Measurement of red blood cell aggregation in a "plate-plate" shearing system by analysis of light transmission. Clin Hemorheol 1998;19:307-14.

15. Baskurt OK, Bor-Küçükatay M, Yalcin O, Meiselman HJ, Armstrong JK. Standard aggregating media to test the "aggregability" of rat red blood cells. Clin Hemorheol Microcirc 2000;22:161-6.

16. Hofstra TC, Kalra VK, Meiselman HJ, Coates TD. Sickle erythrocytes adhere to polymorphonuclear neutrophils and activate the neutrophil respiratory burst. Blood 1996;87:4440-7.

17. Boyum A. Separation of leukocytes from blood and bone marrow. Scand J Clin Lab Invest 1968:(Suppl 97):7.

18. Tsikas D. Analysis of nitrite and nitrate in biological fluids by assays based on the Griess reaction: appraisal of the Griess reaction in the L-arginine/nitric oxide area of research. J Chromatogr B Analyt Technol Biomed Life Sci 2007;851:51-70.

19. Renoe BW, McDonald JM, Ladenson JH. The effects of stasis with and without exercise on free calcium, various cations, and related parameters. Clin Chim Acta 1980; 103:91-100.

20. Nazziola E, House SD. Effects of hydrodynamics and leucocyte-endothelium specificity on leukocyte-endothelium interactions. Microvasc Res 1992;44:127-42.

21. Kuhnle GE, Kuebler WM, Groh J, Goetz AE. Effect of blood flow on leukocyte-endothelium interaction in pulmonary microvessels. Am J Resp Crit Care Med 1995; 152:1221-8.

22. Baskurt OK. Activated granulocyte induced alterations in red blood cells and protection by antioxidant enzymes. Clin Hemorheol 1996;16:49-56.

23. Baskurt OK, Meiselman HJ. Activated polymorphonuclear leukocytes affect red blood cell aggregability. J Leukoc Biol 1998;63:89-93.

24. Baskurt OK, Edremitlioglu M, Temiz A. In vitro effects of in vivo activated leukocytes on RBC filterability and lipid peroxidation. Clin Hemorheol 1994;14:591-6. 
25. Bruckdorfer R. The basics about nitric oxide. Mol Aspects Med 2005;26:3-31.

26. Matsuda N, Hattori Y. Vascular biology in sepsis: pathophysiological and therapeutic significance of vascular dysfunction. J Smooth Muscle Res 2007;43:117-37.

27. Tsui JC, Baker DM, Shaw SG, Dashwood MR. Alterations in nitric oxide synthase isoforms in acute lower limb ischemia and reperfusion. Angiology 2007;58:586-92.

28. Yagi M, Adachi J, Tatsuno Y, Mizuno K. Change in nitric oxide in humans due to application of a pneumatic tourniquet. Clin Chim Acta 1998;278:67-74.
29. Kleinbongard P, Schutz R, Rassaf $T$, Lauer $T$, Dejam A, Jax $T$, et al. Red blood cells express a functional endothelial nitric oxide synthase. Blood 2006;107:294351.

30. Özüyaman B, Grau M, Kelm M, Merx MW, Kleinbongard P. RBC NOS: regulatory mechanisms and therapeutic aspects. Trends Mol Med 2008;14:314-22.

31. Kleinbongard $P$, Dejam A, Lauer T, Rassaf $T$, Schindler A, Picker O, et al. Plasma nitrite reflects constitutive nitric oxide synthase activity in mammals. Free Radic Biol Med 2003;35:790-6. 